\title{
Das Virus und die Toten - Zugangswege zur Geschichte der Spanischen Grippe
}

Wilfried Witte

\footnotetext{
* Die Literaturangaben finden Sie im Internet unter www. saez.ch $\rightarrow$ Aktuelle Nummer oder $\rightarrow$ Archiv $\rightarrow 2010 \rightarrow 21$
}

Korrespondenz:

Dr. med. Wilfried Witte M.A.

Klinik für Anästhesiologie und operative Intensivmedizin, CBF Institut für Geschichte der Medizin, ZHGB Charité - Universitätsmedizin Berlin

Hindenburgdamm 30

D-12203 Berlin

Tel. 00493084452731

wilfried.witte@charite.de
Das öffentliche Interesse an der Geschichte der Grippe unterliegt Konjunkturen. Zwischenzeitlich wird es ruhig um die Grippe und ihre Vergangenheit, bis die Kurve allgemeiner Berichterstattung über Grippe und Grippepandemien vergangener Zeiten wieder stark ansteigt. Journalisten melden sich dann bei Experten. Sie sind vor allem auf der Suche nach Fotos und Filmen aus der Zeit von 1918-1920. Die Bilddokumente, die gesucht werden, sollen ein bestimmtes Sujet bedienen, das die Katastrophe zum Inhalt hat. Es gilt, die grosse Zahl von Toten zu visualisieren. So geschah es zuletzt im Pandemiejahr 2009.

Die Spanische Grippe am Ende des 1. Weltkriegs, die ihren Namen einer fehlerhaften Zuschreibung des Ursprungsorts verdankt, forderte mehr Opfer als der Krieg. Die meisten Grippetoten waren innerhalb von drei bis vier Wochen im Herbst 1918 zu beklagen. Als die gravierendste Grippepandemie der Neuzeit steht sie stets im Mittelpunkt, wenn Grippe historisch beleuchtet wird. Da die Spanische Grippe als Prototyp einer desaströsen Influenzapandemie gilt, die weiterhin medizinische Rätsel aufgibt, konzentriert sich auch das Interesse der Virologie, Demographie und Biomathematik der Grippe historisch auf diese Pandemie.

Die Fragen an die Geschichtsschreibung der Spanischen Grippe oszillieren in Zeiten öffentlichen Interesses am pandemischen Potential der Influenza zwischen zwei Polen: auf der einen Seite der emotionale Bezugspunkt der grossen Zahl der Toten, auf der anderen Seite der rationale Bezugspunkt der virologischen Deutung, die seit der letzten Pandemie jedoch an Gewissheiten ärmer geworden ist.

Wenn man sich abseits dieser polarisierenden Betrachtungsweisen ein Bild von der Grippe-Historiographie machen will, ist es jedoch hilfreich zu wis- sen, wie die Geschichte der Grippe in den vergangenen 90 Jahren geschrieben worden ist.

Der langjährige Grippe-Historiker Howard Phillips aus Kapstadt [1]* hat vor einigen Jahren versucht, die verschiedenen Zugangswege zur Geschichte der Pandemie 1918-1920 zu klassifizieren. Zu unterscheiden sind nach Phillips prinzipiell die Zugangswege als «epidemiology, as high drama, as social science and ecology, and as a scientific saga» [2].

Als der früheste Zugang zur Grippegeschichte kann demnach der epidemiologische Ansatz (epidemiology) angesehen werden. Dabei stehen die geographischen Ursprünge sowie Morbidität, Mortalität und Letalität im Vordergrund. Die bekanntesten Arbeiten stammen von dem Bakteriologen Edwin O. Jordan aus Chicago aus dem Jahre 1927 [3] und von dem Historiker K. David Patterson sowie dem Geographen Gerald Pyle aus dem Jahre 1991 [4].

Im Grossen Drama (high drama) hingegen imponiert die Seuche als Katastrophe, die mitunter romanhaft ausschweifend und klischeehaft, in jedem Fall jedoch in kräftigen Bildern dargestellt wird. Klassisch geworden ist die «Spanish Lady» des historischen Publizisten Richard Collier aus dem Jahre 1974. Die persönlichen Erinnerungen von mehr als 1700 Überlebenden der Grippe, die Colliers Team zusammengetragen hatte, bilden die Grundlage des Buches, das allerdings im Stil von Reader's Digest verfasst worden ist [5]. Auf Deutsch ist zuletzt im vergangenen Jahr eine kurze Gesamtdarstellung der Spanischen Grippe des Historikers und Publizisten Manfred Vasold erschienen. Vasolds Buch ist im Stil des Grossen Dramas verfasst [6]. Darstellungen dieses Stils bedienen am besten den Tagesjournalismus, insofern dieser aus Zeitmangel oder Desinteresse nur die grosse Zahl der Toten abbilden möchte.

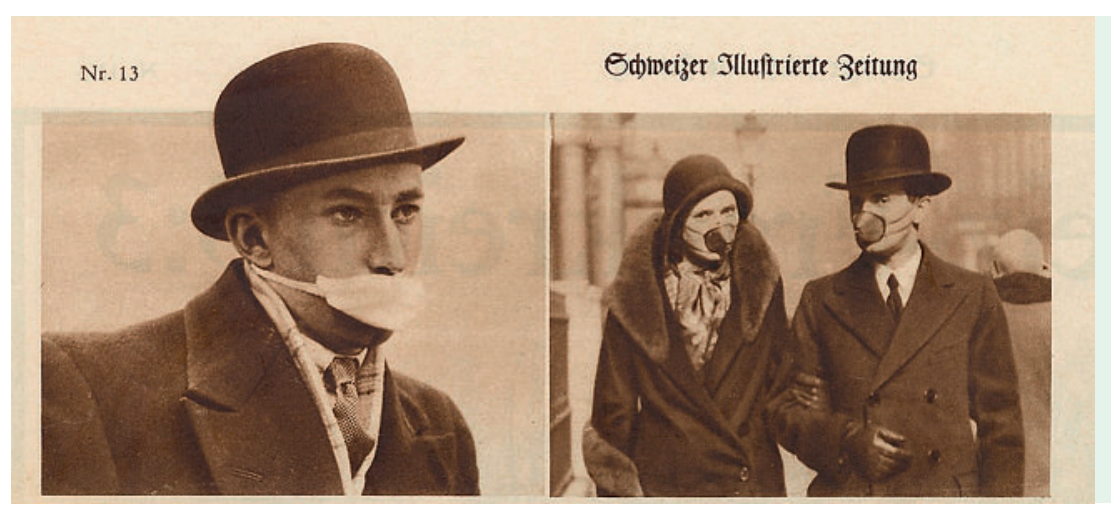

Anti-InfluenzaGesichtsmaske gegen Ansteckung durch die Grippe, wie sie in England empfohlen wurde.

Quelle: Schweizer Illustrierte Zeitung März/ April, ohne Jahresangabe. BSb-Sammlung im Archiv des Medizinhistorischen Instituts der Universität Zürich. 
In sozial- und kulturwissenschaftlicher Perspektive (social science and ecology) wird die Geschichte der Grippe seit den 1970er Jahren untersucht. Den Startschuss dazu hat vor allem Alfred W. Crosby gegeben, dessen Monographie zur Spanischen Grippe in den USA 1976 erstmals erschienen ist [7]. Regionalstudien stehen im Vordergrund. Ihre Anzahl hat Ende des 20. Jahrhunderts stark zugenommen. Gesamtdarstellungen hat es immer wieder gegeben, so ein 550-Seiten-Werk, das in den USA stark nachgefragt worden ist [8], oder ein knappes Bändchen auf Deutsch, dessen Schwergewicht auf den medizinischen Entwicklungen wo sie bislang nur sehr peripher Beachtung gefunden hatte. Der Aufsatz ist jedoch mit dem Makel behaftet, Begriffe wie «Antigen-Drift» und «Antigen-Shift» als historische Wahrheiten zu behandeln, während sie in der Virologie gerade neu bewertet werden. Michels begeht den Fehler, das Virus naiv als historischen Akteur zu benennen. Hiervon ausgehend werden dann vermeintliche Mutationen und Abschwächungen des Virus ex post als erklärend für Entwicklungen an der Westfront gedeutet [16]. Vulgär-Virologie kann jedoch nicht handlungsleitend sein in der Geschichtsschreibung der Spanischen Grippe.

\section{Die Spanische Grippe gilt als Prototyp einer desaströsen Influenza- pandemie, die weiterhin medizinische Rätsel aufgibt}

liegt [9]. Eine Gesamtdarstellung, die den Forschungsstand umfassend wiedergibt, liegt bislang nicht vor [10]. Die Güte der Regionalstudien hängt stark von der Quellenlage $\mathrm{ab}$, die für die angelsächsischen Länder weitaus besser ist als z. B. für das ehemalige Deutsche Reich [11]. Die beste Arbeit über die Grippe in der Schweiz ist nach wie vor eine Berner Lizentiatsarbeit aus dem Jahre 1991 [12].

Die Wissenschaftliche Sage (scientific saga) ist der Zugangsweg der Virologen zum Thema. Ihre eigentlichen Quellen sind gewebliche Überreste ehemaliger Grippe-Opfer, die mit den Mitteln der Molekularbiologie untersucht werden. Prominenteste Vertreter dieser Viroarchäologie sind der US-Amerikaner Jeffrey Taubenberger, dessen Team nach jahrelanger kleinteiliger Arbeit verkündete, das Virus von 1918 rekonstruiert zu haben [13], und der Brite John Oxford, der die These aufgestellt hat, der Ursprung der Spanischen Grippe müsse virologisch in den vorhergehenden Kriegsjahren liegen [14].

Die Viroarchäologie steht immer wieder im Mittelpunkt des Interesses an der Grippe-Geschichte, wenn nicht ihre emotionale, sondern ihre rationale Seite ergründet werden soll. Die Viroarchäologie ist insbesondere durch das Buch der US-amerikanischen Wissenschaftsjournalistin Gina Kolata selbst historisiert worden [15]. Kolata ist es gelungen, die molekularbiologische Entdeckung des Virus vom Herbst 1918 als eine abenteuerliche Story anschaulich zu schildern. Spätestens seit dem Erfolg ihres Buches hat die Neigung, die Geschichte der Spanischen Grippe aus dem Blickwinkel der Virologie zu schildern, stark zugenommen. Virologische Hypothesen und virologisches Lehrbuchwissen, die in der Geschichtsschreibung als Erklärungsansatz verwendet werden, geraten jedoch schnell zu Vulgär-Virologie.

Durch die Publikation seines Habilitationsvortrags hat kürzlich der in London tätige deutsche Historiker Eckard Michels der Spanischen Grippe endgültig den Weg in die deutsche Geschichtswissenschaft gebahnt,
Dieser Fehler unterläuft der Zürcher Wissenschaftshistorikerin Silvia Berger nicht, die kürzlich ihre Dissertation zur Geschichte der medizinischen Bakteriologie 1890-1933 in Deutschland publiziert hat. Bergers Buch beleuchtet die Geschichte der Bakteriologie in einer Weise, die verständlich macht, wie es zum Glauben an die Beherrschbarkeit der Infektionskrankheiten kommen konnte und wie dieser Glaube früh fragwürdig wurde [17]. Die Bakteriologie und frühe Virologie der Grippe spielt in dem Buch allerdings fast keine Rolle.

Bis zum Beweis des Gegenteils sind es die Regionalstudien der «social science and ecology», die die Grippe-Geschichte am ehesten weiterbringen. Als Beleg für diese These kann auch auf die Geschichte Afrikas verwiesen werden. Afrika ist insgesamt von der Spanischen Grippe weitaus stärker betroffen gewesen als Europa. Für die afrikanische Geschichte ist vor einigen Jahren belegt worden, dass eine allein globale Perspektive in der Geschichtsschreibung der Spanischen Grippe dazu angetan ist, die kolonialistische Betrachtung der Seuche auf diesem Kontinent fortzuführen. Konstruktiver dagegen sind auch hier Regionalstudien [18]. Mit ihnen lassen sich Hintergründe und Auswirkungen der Spanischen Grippe am ehesten ausfindig machen.

Es wird wahrscheinlich noch einige Zeit dauern, bis auf dem Boden dieser kleinteiligen Arbeiten grössere Gesamtdarstellungen erscheinen, die die Spanische Grippe angemessen und umfassend dokumentieren. Bis dahin kann man sich darauf verlassen, dass bei ansteigender Grippekonjunktur sich erneut Journalisten bei Experten der Grippegeschichte melden, auf der Suche nach historischem Bildmaterial, das die vielen Grippetoten der Seuche symbolisiert. Die Beiträge schliessen dann weiter nahezu unisono mit der Feststellung, dass die Viroarchäologie die Natur des Virus aufgeklärt habe. Vielleicht setzt sich diese Praxis aber auch unabhängig davon fort, welche Fortschritte die Grippehistoriographie macht. 


\section{Literatur}

1 Phillips H. The Local State and Public Health Reform in South Africa: Bloemfontein and the Consequences of the Spanish Flu Epidemic of 1918. Journal of Southern African Studies. 1987;13:208-233; Phillips H. Black October: the Impact of the Spanish Influenza Epidemic of 1918 on South Africa. Pretoria; 1990.

2 Phillips H. The Re-appearing Shadow of 1918: Trends in the Historiography of the 1918-19 Influenza Pandemic. Canadian Bulletin of Medical History. 2004;21:121-34.

3 Jordan EO. Epidemic Influenza - A Survey. Chicago; 1927.

4 Patterson KD, Pyle GF. The Geography and Mortality of the 1918 Influenza Pandemic. Bulletin of the History of Medicine.1991;65:4-21.

5 Collier R. The Plague of the Spanish Lady. The Influenza Pandemic of 1918-19. London; 1974.

6 Vasold M. Die Spanische Grippe. Die Seuche und der Erste Weltkrieg. Darmstadt; 2009.

7 Taubenberger JK, Reid AH et al. Inititial Genetic Characterization of the 1918 «Spanish» Influenza Virus. Science. 1997;275):1793-6. Taubenberger JK, Reid AH et al. Characterization of the Reconstructed 1918 Spanish Influenza Pandemic Virus. Science. 2005;310:77-80.

8 Oxford JS. World War I may have allowed the emergence of «Spanish» influenza. Infectious Disease 2. The Lancet. 2002;111-4.

9 Witte W. Tollkirschen und Quarantäne. Die Geschichte der Spanischen Grippe. Berlin; 2008, erw. Taschenbuchausgabe; 2010.
10 Den Forschungsstand zu Beginn des 21. Jahrhunderts gibt am besten wieder: Phillips H, Killingray D (Hg.). The Spanish Influenza Pandemic of 1918-19. New Perspectives. London 2003.

11 Siehe hierzu: Witte W. Erklärungsnotstand. Die Grippe-Epidemie 1918-1920 in Deutschland unter besonderer Berücksichtigung Badens. Herbolzheim; 2006. S. 367-85.

12 Sonderegger C. Die Grippeepidemie 1918/19 in der Schweiz. Lizentiatsarbeit. Bern; 1991.

13 Taubenberger JK, Reid AH et al. Initi\$al Genetic Characterization of the 1918 «Spanish» Influenza Virus. Science. 1997;275:1793-6. Taubenberger JK, Reid AH et al. Characterization of the Reconstructed 1918 Spanish Influenza Pandemic Virus. Science. 2005;310:77-80.

14 Oxford JS, World War I may have allowed the emergence of «Spanish» influenza. Infectious Disease 2. The Lancet. 2002:111-4.

15 Kolata G. Flu. The Story of the Great Influenza Pandemic of 1918 and the Search for the Virus that caused it. New; York 1999. Deutsch: Influenza. Die Jagd nach dem Virus. Frankfurt am Main; 2001.

16 Michels E. Die «Spanische Grippe» 1918/19. Verlauf, Folgen und Deutungen in Deutschland im Kontext des Ersten Weltkriegs. Vierteljahreshefte für Zeitgeschichte. 2010;58:1-33.

17 Berger S. Bakterien in Krieg und Frieden. Eine Geschichte der medizinischen Bakteriologie in Deutschland 1890-1933. Göttingen 2009.

18 Heaton M, Falola T. Global Explanations versus Local Interpretations: The Historiography of the Influenza Pandemic of 1918-19 in Africa. History in Africa. 2006;33:205-30. 